\title{
Harnessing the power of mobile phones to improve oral health
}

\section{Sharif M 0, Newton T, Cunningham S J. A systematic review to assess interventions delivered by mobile phones in improving adherence to oral hygiene advice for children and adolescents. Br Dent J 2019: 227: 375-382. https://doi.org/10.1038/s41415-019-0660-5.}

There can be no doubt that mobile phones and smart phones in particular have had a huge impact on our everyday lives. They have positively changed our lives by bringing us almost instant access to huge amounts of information while allowing us to communicate with people across the globe. While they have these benefits they have not been without side-effects and it is common place to see news reports on the pitfalls of social media and mobile phone use. ${ }^{1}$

Healthcare professionals have been quick to see the potential of mobile phones and social media to connect with patients and to influence health behaviours. Mobile devices have been used in a variety of ways from encouraging physical activity and smoking cessation, to monitoring symptoms in conditions such as diabetes and heart disease, or to remind patients of upcoming appointments or to brush their teeth. ${ }^{2}$

With an estimated $78 \%$ of adults possessing a smart phone and two in five people thought to look at their smart phone within five minutes of waking up, ${ }^{3}$ smart phones could hold the potential to positively impact oral health behaviours. One particular area that has been explored is the use of mobile technology to improve oral hygiene in patients through the use of apps such as Brush DJ. ${ }^{4}$

A team based in London has recently undertaken a systematic review, 善 assessing the evidence for the effectiveness of mobile apps to improve otient compliance with oral hygiene advice, in children and adolescent patients. As dental professionals we all know how hard it can be to get patients to change their behaviour, therefore a tool that can help with this () would be greatly beneficial.

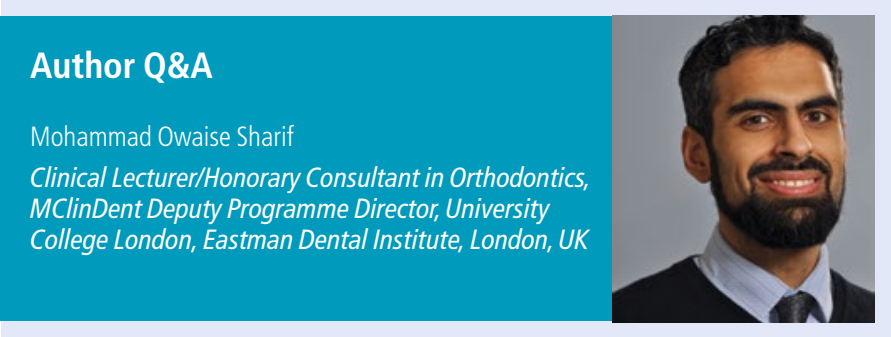

Why did you decide to undertake this study?

I am currently undertaking a $\mathrm{PhD}$ investigating the effectiveness of a personalised mobile phone application in improving adherence to oral hygiene advice amongst orthodontic patients. This systematic review was integral in informing the background of this research and has served to highlight some of the gaps in the current literature.

\section{Did any of the results surprise you?}

Given the availability of mobile phones and apps related to dentistry I was surprised at the lack of published research assessing the effectiveness of dental mobile phone apps aimed at improving oral hygiene.
The review searched for studies involving patients from 10 to 18 years of age. There were two RCT studies, involving 130 orthodontic patients, that met the inclusion criteria, one study from the USA and one from Italy. One of the studies used text message reminders and the other used an app. Patients were followed up for a maximum of 12 months.

The study found that there is moderate evidence for the use of mobile phones in improving adherence to oral health advice with consequent improvements in plaque and bleeding scores.

The authors were only able to identify two RCTs for inclusion and these were in orthodontic patients which does limit the generalisability of the evidence. The study highlights the need for more evidence in this area and the paper highlights that two studies are currently on-going in this area which will improve the evidence further on the effectiveness of mobile phones to improve patient compliance with our oral health advice.

By Kenny Strain, DCT 3

Restorative Department, Leeds Dental Institute

\section{References}

1. Dredge S. Mobile phone addiction? It's time to take back control. The Guardian 2018 Available at https://www.theguardian.com/technology/2018/jan/27/mobile-phone-addiction-apps-break-the-habit-take-back-control (accessed August 2019).

2. Klasnja P, Pratt W. Healthcare in the pocket: Mapping the space of mobile-phone health interventions. J Biomed Inform 2012; 45: 184-198.

3. Wakefield J. Phone and internet use: Number of mobile calls drops for first time. BBC News, 2018. Available at https://www.bbc.co.uk/news/technology-45033302 (accessed August 2019).

4. Underwood B. Brush DJ. Available at http://www.brushdj.com/\#nogo (accessed August 2019).

What do you think the next steps should be considering your findings? In the short term there is clearly a need to assess the quality (including knowledge content) and effectiveness of the available dental mobile phone apps aimed at patients. Given that the number of available apps is significantly greater than those that have been robustly assessed for effectiveness, this approach will help dental professionals navigate patients towards the most useful resources. In the medium to long term, there is a need to develop mobile phone interventions that are grounded in behaviour change theory and contain appropriate behaviour change theories. The available evidence shows that interventions based on behaviour change theory and those with more behaviour change techniques (BCTs) are more effective than those that are not based on theory and with fewer BCTs. Future studies designed to assess the effectiveness of such interventions should utilise a core outcome set related to oral hygiene and explore outcomes related to patient satisfaction and engagement with the technologies being tested - this may help to identify features of successful digital interventions. The utilisation of a core outcome set would also increase the likelihood of a meta-analysis being possible. 